\title{
SISTEMA FUZZY PARA PREDIÇÃO DO DESEMPENHO PRODUTIVO DE FRANGOS DE CORTE DE 1 A 21 DIAS DE IDADE
}

\section{PATRÍCIA F. PONCIANO ${ }^{1}$, TADAYUKI YANAGI JUNIOR ${ }^{2}$, LEONARDO SCHIASSI ${ }^{3}$, ALESSANDRO T. CAMPOS ${ }^{4}$, JOSÉ W. B. DO NASCIMENTO $^{5}$}

\begin{abstract}
RESUMO: Um sistema de inferência fuzzy foi desenvolvido baseado em dados da literatura para predição do consumo de ração, ganho de peso e conversão alimentar de frangos de corte com idade variando de 1 a 21, dias submetidos a diferentes condições térmicas. O sistema fuzzy foi estruturado com base em três variáveis de entrada: idade das aves (semanas), temperatura $\left({ }^{\circ} \mathrm{C}\right)$ e umidade relativa (\%) ambientes, sendo que as variáveis de saída consideradas foram: ganho de peso, consumo de ração e conversão alimentar. A inferência foi realizada por meio do método de Mamdani, que consistiu na elaboração de 45 regras e a defuzzificação por meio do método do Centro de Gravidade. Com base nos resultados, ao se compararem os dados da literatura com os obtidos pelo sistema fuzzy proposto, verificou-se desempenho satisfatório na predição das variáveis respostas, com $\mathrm{R}^{2}$ da ordem de 0,995; 0,998 e 0,976, respectivamente. O ganho de peso predito pela lógica fuzzy foi validado com dados experimentais de campo, no qual se obteve $\mathrm{R}^{2}=0,975$, apresentando grande potencial de uso em sistemas de climatização automatizado.
\end{abstract}

PALAVRAS-CHAVE: modelagem, inferência fuzzy, conforto ambiental.

\section{FUZZY SYSTEM TO PREDICT PRODUCTIVE PERFORMANCE OF BROILER CHICKS FROM 1 TO 21 DAYS OLD}

\begin{abstract}
A fuzzy inference system was developed based on literature data to predict feed intake, weight gain, and feed conversion of broiler chicks from 1 to 21 day old submitted to different thermal conditions. The fuzzy system was structured based on three input variables: age of chick (weeks), ambient temperature $\left({ }^{\circ} \mathrm{C}\right)$ and ambient relative humidity (\%); and the output variables considered were: weight gain, feed intake and feed conversion. The inference was performed using the Mamdani's method, which consisted of the elaboration of 45 rules, and the defuzzification using the Center of Gravity method. Comparing literature data with the results obtained by the fuzzy system proposed, it is possible to conclude that the fuzzy system predicts satisfactorily the weight gain, feed intake and feed conversion, which $\mathrm{R}^{2}$ were $0.995,0.998$, and 0.976 , respectively. The weight gain predicted by the fuzzy system was validated by experimental field data, which obtained $\mathrm{R}^{2}=0.9753$, presenting a considerable use potential in automatic acclimatization systems.
\end{abstract}

KEYWORDS: modeling, fuzzy inference, environmental comfort.

\section{INTRODUÇÃO}

Dentre os desafios enfrentados pela avicultura de corte, destaca-se a importância do ambiente de criação. Sabe-se que o sistema de criação intensivo possui influência direta na condição de conforto e bem-estar animal, promovendo dificuldade na manutenção do balanço térmico, no interior das instalações e na expressão dos comportamentos naturais dos animais, afetando o desempenho produtivo das aves (MOURA et al., 2006; SALGADO et al., 2007; NAZARENO et al., 2009; VIGODERIS et al., 2010). Entre os fatores ambientais, os elementos meteorológicos,

\footnotetext{
${ }^{1}$ Zootecnista, Doutoranda em Engenharia Agrícola, Universidade Federal de Lavras, Departamento de Engenharia , Lavras - MG.

${ }^{2}$ Prof. Associado da Universidade Federal de Lavras, Departamento de Engenharia, Lavras - MG.

${ }^{3}$ Eng ${ }^{\circ}$ Agrícola, Doutorando em Engenharia Agrícola, Universidade Federal de Lavras, Departamento de Engenharia, Lavras - MG.

${ }^{4}$ Prof. Adjunto, Universidade Federal de Lavras, Departamento de Engenharia, Lavras - MG.

${ }^{5}$ Prof. Associado, Universidade Federal de Campina Grande, Unidade Acadêmica de Engenharia Agrícola, Campina Grande - PB.

Recebido pelo Conselho Editorial em: 17-8-2011

Aprovado pelo Conselho Editorial em: 3-2-2012
} 
representados, principalmente, pela temperatura e umidade relativa do ar, são os que afetam diretamente as aves, pois comprometem a manutenção da homeotermia (OLIVEIRA et al., 2006).

Dados de conforto térmico para pintinhos têm sido muito citados na literatura e mostram que tanto o estresse por calor, quanto aquele pelo frio durante as primeiras três semanas de vida podem causar perda de peso corporal além de outros prejuízos para a saúde da ave (MOURA et al., 2008). Após a eclosão, o desenvolvimento inicial do pintinho é fundamental para o melhor desempenho do frango de corte até o final do ciclo de produção (TEIXEIRA et al., 2009). O pintinho, nos primeiros dias após a eclosão, é considerado um animal pecilotérmico, ou seja, sua temperatura corporal sofre variações de acordo com a temperatura ambiente. Isto ocorre porque essas aves ainda não têm seu sistema termorregulador amadurecido, nem reserva energética suficiente para serem capazes de se adaptar às condições adversas do ambiente.

O desenvolvimento do pintinho em condições ambientais de termoneutralidade, em particular na primeira semana de idade, é condição relevante para o desenvolvimento futuro do animal (MARCHINI et al., 2009). Usualmente, os limites de termoneutralidade são fixados em medidas indiretas, como temperatura do ar, umidade relativa, ventilação, entre outras variáveis ambientais. Entretanto, para MENEZES et al. (2010), a T e a UR foram identificadas como sendo alguns dos pontos críticos de controle e possíveis riscos na criação de frangos. Segundo OLIVEIRA et al. (2006), a temperatura adequada para criação de pintinhos é de 32 a $34{ }^{\circ} \mathrm{C}, 28$ a $32{ }^{\circ} \mathrm{C}$ e de 26 a $28{ }^{\circ} \mathrm{C}$ na primeira, segunda e terceira semanas de vida, respectivamente. A umidade relativa, nos primeiros dias de vida, deve manter-se por volta de 70\%, posteriormente, a umidade relativa deve manter-se entre 50-60\% (JACOMÉ et al., 2007). Segundo MANAGEMENT GUIDE BROILER (2006), de maneira geral, a faixa de velocidade do ar, que contempla um maior desempenho para pintinhos de 1 a 21, dias deve ser menor que $0,1 \mathrm{~m} \mathrm{~s}^{-1}$, ou seja, quase nula.

Assim, a estimativa de bem-estar animal constitui-se em um problema em que a aplicação da teoria dos conjuntos fuzzy pode apresentar-se promissora (OWADA et al., 2007).

A lógica fuzzy, dentre outras ferramentas direcionadas para a tomada de decisão e para ações mais precisas, têm contribuído para o avanço e a velocidade das pesquisas em ambiência e produção animal (CADENAS et al., 2004; PANDORFI et al., 2007; PEREIRA et al., 2008; NÄÄS et al., 2008; PERISSINOTTO et al., 2009; SANTOS et al., 2009; TOLON et al., 2010). Dentre estes trabalhos, destaca-se a utilização da lógica fuzzy como suporte à tomada de decisão nas áreas de ambiência e produção avícola.

Diante da versatilidade apresentada por esta ferramenta matemática computacional, o presente trabalho teve o objetivo de desenvolver um modelo fuzzy para predição do consumo de ração, ganho de peso e conversão alimentar de frangos de corte com idade variando de 1 a 21 dias.

\section{MATERIAL E MÉTODOS}

\section{Desenvolvimento do modelo fuzzy}

O modelo fuzzy foi elaborado com base em dados experimentais oriundos da literatura, sobre o desempenho produtivo de pintinhos das linhagens Cobb com idade entre 1 a 21 dias, expostos a diferentes condições térmicas.

Para cada variável, foram atribuídos conjuntos fuzzy que as caracterizaram, sendo que, para cada conjunto fuzzy, foi criada uma função de pertinência. Neste trabalho, foram utilizadas como variáveis de entrada, a idade das aves (I, dias), chamada de idade 1 [ 1 a 8 dias], idade 2 [7 a 15 dias] e idade 3 [14 a 21 dias], e a temperatura do ar $\left(\mathrm{T},{ }^{\circ} \mathrm{C}\right)$, que foram classificadas em $\mathrm{T} 1$ [23;26]; T2 [25;28,5]; T3 [28;30]; T4 [29;33] e T5 [32;35]; e a umidade relativa do ar (UR, \%) que foi classificada em baixa [30 a 58\%], adequada [55 a 75\%] e alta [70 a 90\%]. Fez-se uso de variáveis linguísticas genéricas para a temperatura porque os 5 intervalos foram utilizados para as 3 semanas de vida das aves. Dessa forma, não existe um nivel de temperatura que pode ser considerado ideal para as 3 semanas de vida, simultaneamente. Pois cada idade tem um intervalo de temperatura 
considerado mais adequado. As variáveis de saída foram o ganho de peso (GP, g), o consumo de ração $(\mathrm{CR}, \mathrm{g})$ e a conversão alimentar $\left(\mathrm{CA}, \mathrm{g} \mathrm{g}^{-1}\right)$ de pintinhos do $1^{\mathrm{o}}$ até o $21^{\circ}$ dia de vida.

Os intervalos admitidos para as variáveis de entrada (I, T, UR) foram listados na Tabela 1 e representados por curvas de pertinência trapezoidais (Figura 1), pelo fato de representarem melhor as classes dos dados de entrada, solução encontrada por vários autores como AMENDOLA et al. (2004); OLIVEIRA et al. (2005), PANDORFI et al. (2007), e TOLON et al. (2010). As classificações adotadas foram baseadas na literatura, no ajuste entre os valores simulados e observados, e na consulta a especialistas.

TABELA 1. Conjuntos fuzzy para as variáveis de entrada. Fuzzy sets for the input variables.

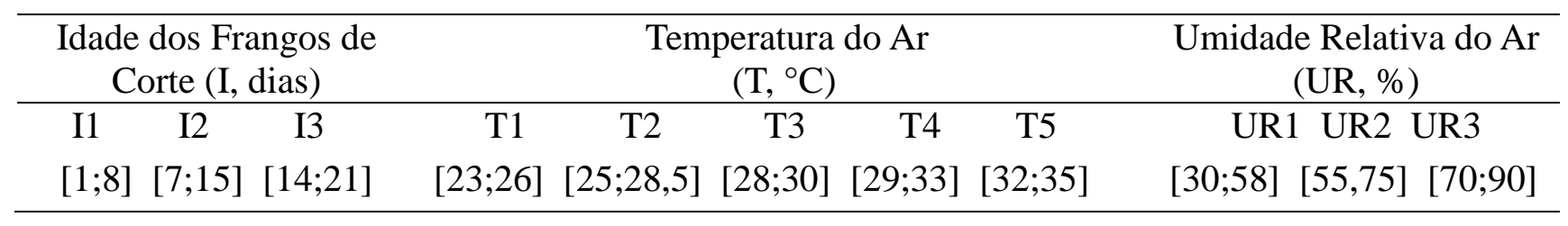

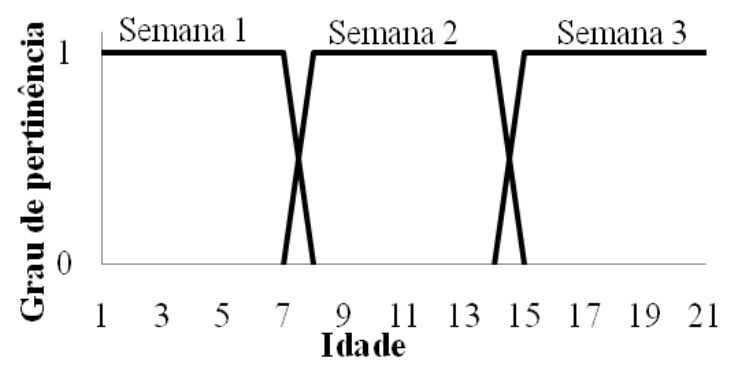

(a)

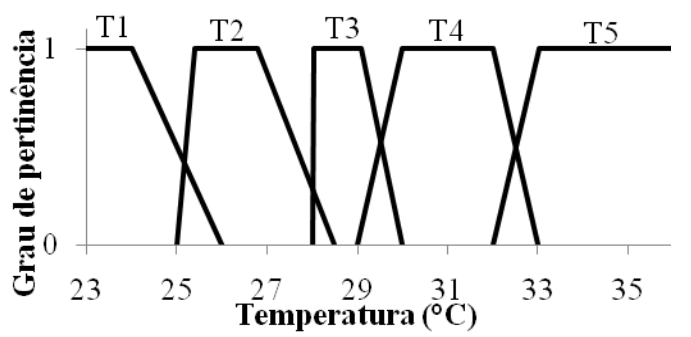

(b)

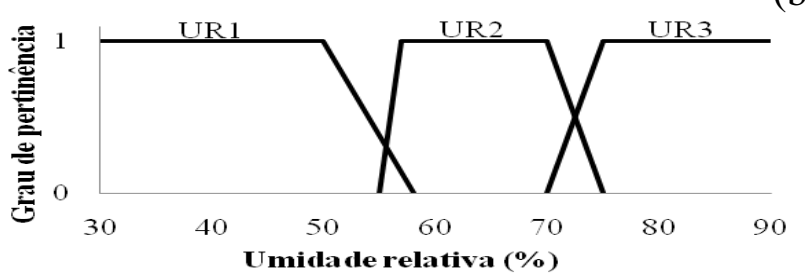

(c)

FIGURA 1. Curvas de pertinência das variáveis de entrada da lógica fuzzy: (a) Idade do frango de corte, (b) Temperatura $\left({ }^{\circ} \mathrm{C}\right)$; (c) Umidade relativa (\%). Pertinence functions of the fuzzy sets considered as input: (a) Chickens'age, (b) Temperature $\left({ }^{\circ} \mathrm{C}\right)$, (c) Relative humidity (\%).

Para o desenvolvimento do modelo fuzzy, selecionaram-se três especialistas, oriundos de grupos heterogêneos, com experiência na área de ambiência animal e conhecimentos teóricos obtidos por meio de pesquisas científicas e ministração de disciplinas, de cursos e de palestras relacionadas ao assunto, conforme metodologia de seleção de especialista fuzzy proposta por CORNELISSEN et al. (2002). Segundo AYYUB \& KLIR (2006), o especialista é uma pessoa que possui conhecimento profundo sobre determinado assunto; desta forma, selecionaram-se especialistas que possuíam mais de 10 anos de experiência na área citada previamente. Ademais, o número de especialistas selecionados atende à quantidade proposta por KLIR \& YUAN (1995), que é de um ou mais. A adequada seleção de especialistas interfere na confiabilidade dos resultados (TAYLOR, 1988); desta forma, ela foi verificada por meio do ajuste entre valores simulados e medidos.

As curvas de pertinência para as variáveis de saída (Figura 2) foram desenvolvidas com base nas pesquisas conduzidas por SANTOS (2004), CARVALHO (2006), MINAFRA (2007), LABOISSIÈRE (2008), LIRA et al. (2009), THON et al. (2010) e ZAUK (2008), que forneceram informações a respeito das respostas produtivas nas 3 semanas iniciais de vida, 5 classes de 
temperatura e 3 umidades relativas, totalizando 45 regras, que contemplam as condições necessárias paras as 3 semanas de vida.

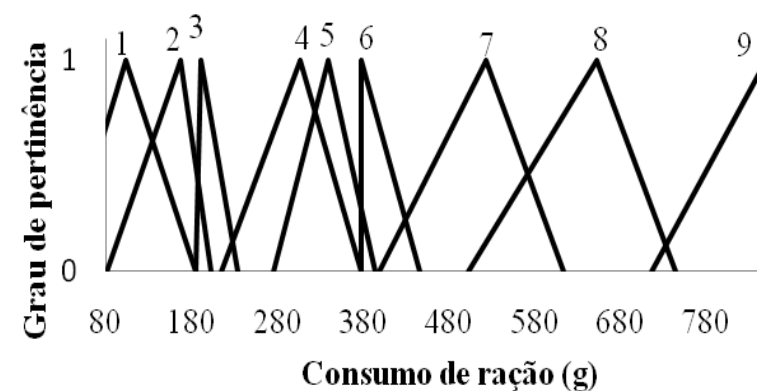

(a)

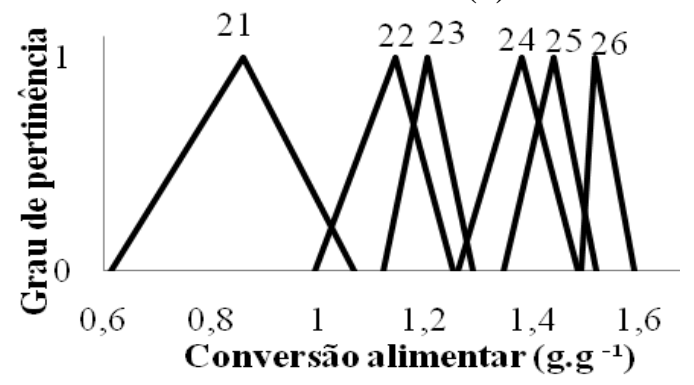

(c)

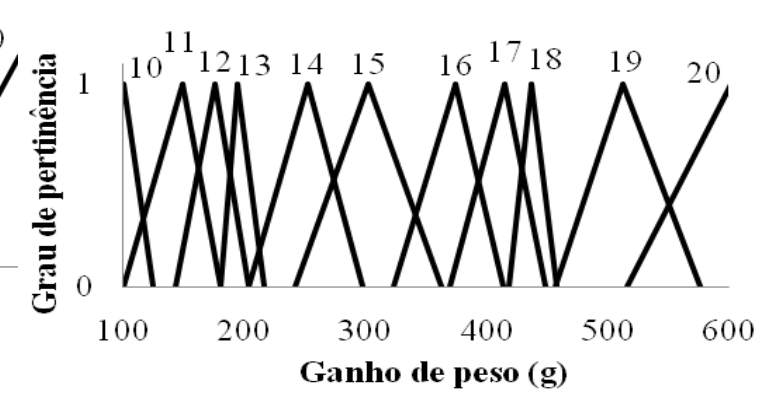

(b)

\begin{tabular}{|ll|}
\hline \multicolumn{2}{|c|}{ Legenda } \\
\hline 1 a 9 & Curvas de pertinência do CR \\
10 a 20 & Curvas de pertinência do GP \\
21 a 26 & Curvas de pertinência da CA \\
\hline
\end{tabular}

FIGURA 2. Curvas de pertinência para as variáveis de saída: (a) consumo de ração (g); (b) ganho de peso $(\mathrm{g})$, e (c) conversão alimentar $\left(\mathrm{g} \mathrm{g}^{-1}\right)$. Pertinence functions of the fuzzy sets considered as output: (a) feed intake (g), (b) weight gain (g) and (c) feed conversion $\left(\mathrm{g} \mathrm{g}^{-1}\right)$.

Os intervalos adotados para as variáveis de saída (GP, CR, CA) foram caracterizados por curvas de pertinência do tipo triangular (Figura 2), por representarem melhor o conjunto de dados e por resultarem nos menores valores de desvio-padrão, solução adotada por diversos autores (OWADA et al., 2007; PEREIRA et al., 2008; SANTOS et al., 2009).

O método de inferência utilizado foi o de Mamdani, que também foi utilizado por AMENDOLA et al. (2005) e TOLON et al. (2010). Esse método traz como resposta um conjunto fuzzy originado da combinação dos valores de entrada com os seus respectivos graus de pertinência, por meio do operador mínimo e, em seguida, pela superposição das regras pelo operador máximo.

A defuzzificação realizou a tradução do valor linguístico em valor numérico, que foi feita utilizando-se do método do Centro de Gravidade, que considera todas as possibilidades de saída, transformando o conjunto fuzzy, originado pela inferência, em valor numérico, conforme proposto por AMENDOLA et al. (2005).

A base de regras (Tabela 2) foi composta por uma coleção de proposições fuzzy, apresentadas na forma SE-E-ENTÃO, tendo sido elaborada a partir de informações da Tabela 1 e com o auxílio de especialistas da área. A base foi composta por 45 regras, sendo que, para cada regra, foi atribuído um fator de ponderação igual a 1 . O ambiente computacional utilizado foi o MAPLE ${ }^{\circledR} 13$.

\section{Validação do modelo fuzzy}

A validação do modelo fuzzy foi feita por meio de experimento de campo realizado numa granja comercial com frangos machos da linhagem Cobb, no município de São Sebastião do Oeste - MG, situado na latitude $20^{\circ} 16^{\prime} 33^{\prime \prime} \mathrm{S}$ e longitude $45^{\circ} 00^{\prime} 18^{\prime \prime} \mathrm{O}$, altitude $780 \mathrm{~m}$, no período de 28 de setembro a 19 de outubro de 2010. Utilizaram-se 2 galpões estruturalmente idênticos, orientados na direção nordeste - sudoeste, com $160 \mathrm{~m}$ de comprimento e $13 \mathrm{~m}$ de largura; 2,45 m de pé-direito, cobertura de telhas de cimento- amianto de $4 \mathrm{~mm}$ de espessura. 
TABELA 2. Sistema de regras da inferência fuzzy para as características idade das aves (I), temperatura (T) e umidade relativa (UR). Rules' system used in fuzzy inference for the characteristics chickens' age (I), temperature (T) and relative humidity (RH).

\begin{tabular}{|c|c|}
\hline & Regra \\
\hline 1 & SE I é I1 E T é T1 E UR é UR1 ENTÃO CR é CR1, GP é GP1 e CA é CA2 \\
\hline 2 & SE I é I1 E T é T1 E UR é UR2 ENTÃO CR é CR1, GP é GP1 e CA é CA2 \\
\hline 3 & SE I é I1 E T é T1 E UR é UR3 ENTÃO CR é CR1, GP é GP1 e CA é CA2 \\
\hline 4 & SE I é I1 E T é T2 E UR é UR1 ENTÃO CR é CR1, GP é GP1 e CA é CA2 \\
\hline 5 & SE I é I1 E T é T2 E UR é UR2 ENTÃO CR é CR1, GP é GP1 e CA é CA2 \\
\hline 6 & SE I é I1 E T é T2 E UR é UR3 ENTÃO CR é CR2, GP é GP1 e CA é CA2 \\
\hline 7 & SE I é I1 E T é T3 E UR é UR1 ENTÃO CR é CR2, GP é GP4 e CA é CA1 \\
\hline 8 & SE I é I1 E T é T3 E UR é UR2 ENTÃO CR é CR2, GP é GP1 e CA é CA2 \\
\hline 9 & SE I é I1 E T é T3 E UR é UR3 ENTÃO CR é CR3, GP é GP3 e CA é CA2 \\
\hline 10 & SE I é I1 E T é T4 E UR é UR1 ENTÃO CR é CR1, GP é GP2 e CA é CA1 \\
\hline 11 & SE I é I1 E T é T4 E UR é UR2 ENTÃO CR é CR1, GP é GP1 e CA é CA2 \\
\hline 12 & SE I é I1 E T é T4 E UR é UR3 ENTÃO CR é CR2, GP é GP2 e CA é CA2 \\
\hline 13 & SE I é I1 E T é T5 E UR é UR1 ENTÃO CR é CR2, GP é GP2 e CA é CA2 \\
\hline 14 & SE I é I1 E T é T5 E UR é UR2 ENTÃO CR é CR2, GP é GP2 e CA é CA2 \\
\hline 15 & SE I é I1 E T é T5 E UR é UR3 ENTÃO CR é CR2, GP é GP2 e CA é CA2 \\
\hline 16 & SE I é I2 E T é T1 E UR é UR1 ENTÃO CR é CR4, GP é GP4 e CA é CA3 \\
\hline 17 & SE I é I2 E T é T1 E UR é UR2 ENTÃO CR é CR5, GP é GP5 e CA é CA5 \\
\hline 18 & SE I é I2 E T é T1 E UR é UR3 ENTÃO CR é CR4, GP é GP4 e CA é CA2 \\
\hline 19 & SE I é I2 E T é T2 E UR é UR1 ENTÃO CR é CR4, GP é GP4 e CA é CA4 \\
\hline 20 & SE I é I2 E T é T2 E UR é UR2 ENTÃO CR é CR4, GP é GP5 e CA é CA3 \\
\hline 21 & SE I é I2 E T é T2 E UR é UR3 ENTÃO CR é CR4, GP é GP5 e CA é CA3 \\
\hline 22 & SE I é I2 E T é T3 E UR é UR1 ENTÃO CR é CR4, GP é GP5 e CA é CA3 \\
\hline 23 & SE I é I2 E T é T3 E UR é UR2 ENTÃO CR é CR4, GP é GP5 e CA é CA4 \\
\hline 24 & SE I é I2 E T é T3 E UR é UR3 ENTÃO CR é CR7, GP é GP7 e CA é CA4 \\
\hline 25 & SE I é I2 E T é T4 E UR é UR1 ENTÃO CR é CR6, GP é GP6 e CA é CA4 \\
\hline 26 & SE I é I2 E T é T4 E UR é UR2 ENTÃO CR é CR5, GP é GP6 e CA é CA2 \\
\hline 27 & SE I é I2 E T é T4 E UR é UR3 ENTÃO CR é CR5, GP é GP5 e CA é CA5 \\
\hline 28 & SE I é I2 E T é T5 E UR é UR1 ENTÃO CR é CR4, GP é GP4 e CA é CA5 \\
\hline 29 & SE I é I2 E T é T5 E UR é UR2 ENTÃO CR é CR3, GP é GP4 e CA é CA5 \\
\hline 30 & SE I é I2 E T é T5 E UR é UR3 ENTÃO CR é CR3, GP é GP4 e CA é CA5 \\
\hline 31 & SE I é I3 E T é T1 E UR é UR1 ENTÃO CR é CR7, GP é GP8 e CA é CA5 \\
\hline 32 & SE I é I3 E T é T1 E UR é UR2 ENTÃO CR é CR8, GP é GP8 e CA é CA6 \\
\hline 33 & SE I é I3 E T é T1 E UR é UR3 ENTÃO CR é CR8, GP é GP8 e CA é CA6 \\
\hline 34 & SE I é I3 E T é T2 E UR é UR1 ENTÃO CR é CR7, GP é GP7 e CA é CA5 \\
\hline 35 & SE I é I3 E T é T2 E UR é UR2 ENTÃO CR é CR5, GP é GP6 e CA é CA5 \\
\hline 36 & SE I é I3 E T é T2 E UR é UR3 ENTÃO CR é CR9, GP é GP11 e CA é CA5 \\
\hline 37 & SE I é I3 E T é T3 E UR é UR1 ENTÃO CR é CR8, GP é GP9 e CA é CA5 \\
\hline 38 & SE I é I3 E T é T3 E UR é UR2 ENTÃO CR é CR9, GP é GP6 e CA é CA5 \\
\hline 39 & SE I é I3 E T é T3 E UR é UR3 ENTÃO CR é CR8, GP é GP6 e CA é CA5 \\
\hline 40 & SE I é I3 E T é T4 E UR é UR1 ENTÃO CR é CR8, GP é GP10 e CA é CA6 \\
\hline 41 & SE I é I3 E T é T4 E UR é UR2 ENTÃO CR é CR8, GP é GP8 e CA é CA6 \\
\hline 42 & SE I é I3 E T é T4 E UR é UR3 ENTÃO CR é CR7, GP é GP7 e CA é CA6 \\
\hline 43 & SE I é I3 E T é T5 E UR é UR1 ENTÃO CR é CR7, GP é GP7 e CA é CA6 \\
\hline 44 & SE I é I3 E T é T5 E UR é UR2 ENTÃO CR é CR7, GP é GP6 e CA é CA6 \\
\hline 45 & SE I é I3 E T é T5 E UR é UR3 ENTÃO CR é CR6, GP é GP6 e CA é CA6 \\
\hline
\end{tabular}


O registro de temperatura (T) e de umidade relativa do ar (UR) foi feita a cada 5 min, durante os primeiros 21 dias de idade das aves, por meio de sensores registradores de temperatura e umidade relativa modelo Hobo Pro Series, Onset ${ }^{\circledR}$, com precisão de $\pm 3 \%$ da leitura. Os dois galpões de frangos foram divididos ao meio, de forma que os pintinhos ficassem separados entre os lados nordeste e sudoeste do galpão sem, no entanto, haver diferença alguma no manejo realizado entre os dois lados. Em cada lado do galpão, havia a contenção que restringia o espaço disponível para as aves. A cada dois dias, essa contenção era movida e aumentava o espaço disponível para as aves. No $18^{\circ}$ dia, elas passaram a ocupar o galpão todo. Os sensores/registradores de T e UR acompanhavam essa movimentação da contenção, sendo que L representa a largura e C o comprimento (Figura 3).

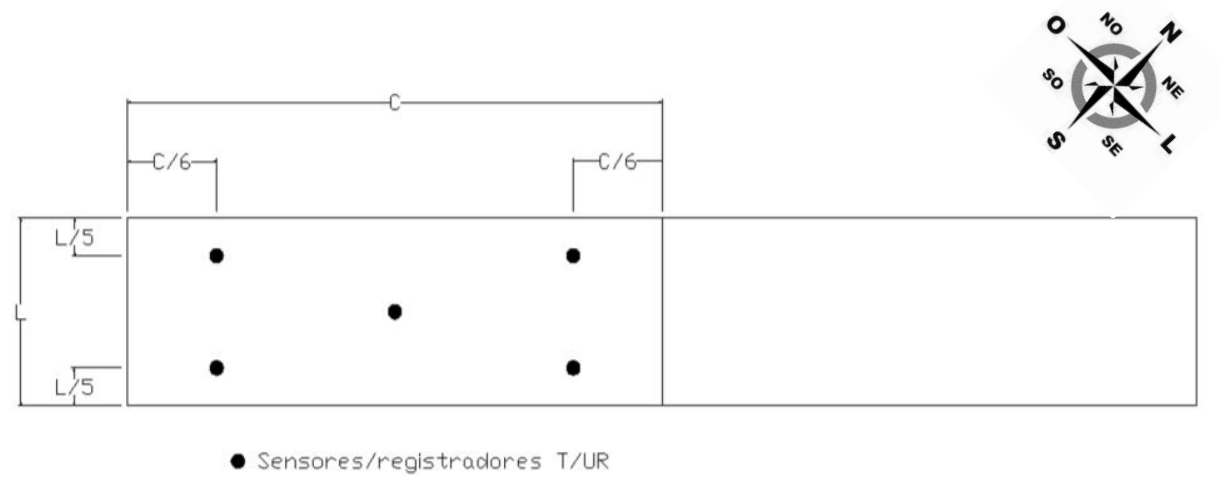

FIGURA 3. Esquema de posicionamento dos sensores/registradores de temperatura e umidade relativa nos galpões de frango. Positioning scheme of dataloggers of temperature and relative humidity in chicken houses.

Diariamente, a massa corporal de 60 pintinhos, em cada galpão (120 aves ao todo), era avaliada para que fosse feito o acompanhamento do ganho de massa do lote. As medições de CR e CA não foram feitas devido à impossibilidade de se alterar o manejo da granja; entretanto, foram mantidas devido a sua importância e por existirem dados da literatura que possibilitaram a verificação do modelo.

Durante a fase experimental, as aves tiveram acesso à água na primeira semana por meio de 4 linhas de bebedouros pendulares, e a partir da segunda semana por 5 linhas, com espaçamento de 2,0 $\mathrm{m}$ e cerca de 100 aves por bebedouro. Na primeira semana, a ração foi distribuída por meio de 1 linha de comedouros automáticos, com espaçamento de $0,65 \mathrm{~m}$ e 5 linhas de comedouros manuais distribuídos aleatoriamente. Já na segunda semana, foram utilizadas 3 linhas de comedouros automáticos e 7 linhas de comedouros manuais. A partir do $18^{0}$ dia, foram removidos os comedouros manuais, deixando apenas os automáticos.

As dietas fornecidas aos animais foram formuladas de acordo com as recomendações do National Research Council-NRC (1994), com base nas exigências de nutrientes para as diferentes fases de crescimento e foram iguais para todos os sistemas.

\section{RESULTADOS E DISCUSSÃO}

De acordo com CORDEIRO et al. (2010), as primeiras semanas de vida da ave são as mais críticas, e erros cometidos nesta fase não poderão ser corrigidos a contento no futuro, e isto fará com que o desempenho final das aves seja afetado. Daí a importância de se adaptar o ambiente às condições ideais de bem-estar para as aves mais jovens. De acordo com OLIVEIRA et al. (2006), a temperatura para a faixa de conforto, na primeira semana de vida das aves, situa-se entre 32 a $34{ }^{\circ} \mathrm{C}$. Entretanto, o máximo $\mathrm{CR}$ e $\mathrm{GP}$ e a melhor $\mathrm{CA}$ foram alcançados na condição de aproximadamente $29^{\circ} \mathrm{C}$ (Tabela 3). LIRA et al. (2009) justificam que essas respostas das aves nestas semanas podem ocorrer pela tentativa de adaptação fisiológica à situação de menor conforto. 
ABREU et al. (2000) afirmam que aves submetidas a baixas temperaturas podem aumentar seu CR, porém isto não implica melhoria da taxa de crescimento, uma vez que parte da energia ingerida é utilizada especificamente para a produção de calor. No experimento realizado por SILVA et al. (2009), a temperatura alta na fase de 1 a 7 dias de idade piorou a CA, que apresentou os melhores valores nas temperaturas controle e baixa. $\mathrm{Na}$ segunda semana de vida, a faixa de temperatura de conforto térmico recomendada pelos mesmos autores, citados previamente, está entre 28 a $32{ }^{\circ} \mathrm{C}$, e na terceira semana, entre 26 a $28^{\circ} \mathrm{C}$.

TABELA 3. Comparação dos valores de consumo de ração (CR), ganho de peso (GP) e conversão alimentar (CA) obtida por meio de dados experimentais oriundos da literatura (ME) e sistema fuzzy (SF). Comparison of feed intake (CR), weight gain (GP) and feed conversion (CA) obtained by experimental data from literature (ME) and fuzzy system (SF).

\begin{tabular}{|c|c|c|c|c|c|c|c|c|c|c|c|c|c|c|c|c|}
\hline \multirow[b]{2}{*}{ Sem } & \multirow[b]{2}{*}{$\begin{array}{c}\mathrm{T} \\
\left({ }^{\circ} \mathrm{C}\right)\end{array}$} & \multirow[b]{2}{*}{$\begin{array}{l}\text { UR } \\
(\%)\end{array}$} & \multicolumn{3}{|c|}{ ME } & \multicolumn{2}{|c|}{ SF } & \multicolumn{3}{|c|}{ Desvio-Padrão } & \multicolumn{3}{|c|}{ Desvio Absoluto } & \multicolumn{3}{|c|}{ Erro $(\%)$} \\
\hline & & & $\mathrm{CR}$ & GP & $\mathrm{CA}$ & CR GP & $\mathrm{CA}$ & $\mathrm{CR}$ & GP & $\mathrm{CA}$ & $\mathrm{CR}$ & GP & $\mathrm{CA}$ & $\mathrm{CR}$ & GP & $\mathrm{CA}$ \\
\hline \multirow{8}{*}{1} & $24,3^{\mathrm{a}}$ & $55,8^{\mathrm{a}}$ & $114^{\mathrm{a}}$ & $101^{\mathrm{a}}$ & $1,13^{\mathrm{a}}$ & 123109 & 1,13 & 6,36 & 5,66 & 0,000 & 9,00 & 8,00 & 0,00 & 7,89 & 7,92 & 0,00 \\
\hline & $26,8^{\mathrm{b}}$ & $53,1^{b}$ & $124,5^{\mathrm{b}}$ & $114,4^{\mathrm{b}}$ & $1,09^{\mathrm{b}}$ & 119108 & 1,13 & 3,89 & 4,53 & 0,029 & 5,50 & 6,40 & 0,04 & 4,42 & 5,59 & 3,76 \\
\hline & $27,85^{\mathrm{c}}$ & $52,3^{\mathrm{c}}$ & $133^{\mathrm{c}}$ & $112^{\mathrm{c}}$ & $1,19^{\mathrm{c}}$ & 123109 & 1,13 & 7,07 & 2,12 & 0,042 & 10,00 & 3,00 & 0,06 & 7,52 & 2,68 & 5,04 \\
\hline & $28,91^{\mathrm{d}}$ & $78^{\mathrm{d}}$ & $199,2^{\mathrm{d}}$ & $173,3^{\mathrm{d}}$ & $1,1^{\mathrm{d}}$ & 203174 & 1,13 & 2,71 & 0,49 & 0,021 & 3,83 & 0,69 & 0,03 & 1,92 & 0,40 & $2,, 73$ \\
\hline & $28,91^{\mathrm{d}}$ & $78^{\mathrm{d}}$ & $199,3^{\mathrm{d}}$ & $177,8^{\mathrm{d}}$ & $1,13^{\mathrm{d}}$ & 203174 & 1,13 & 2,62 & 2,68 & 0,000 & 3,71 & 3,79 & 0,00 & 1,86 & 2,13 & 0,00 \\
\hline & $29^{\mathrm{e}}$ & $51^{\mathrm{e}}$ & $158,5^{\mathrm{e}}$ & $198,1^{\mathrm{e}}$ & $0,8^{\mathrm{e}}$ & 150198 & 0,847 & 5,98 & 0,05 & 0,033 & 8,46 & 0,07 & 005 & 5,34 & 0,04 & 5,87 \\
\hline & $29,5^{\mathrm{e}}$ & $37^{\mathrm{e}}$ & $139,9^{\mathrm{e}}$ & $171,3^{\mathrm{e}}$ & $0,89^{\mathrm{e}}$ & 136161 & 0,845 & 2,74 & 7,30 & 0,032 & 3,87 & 10,33 & 0,05 & 2,77 & 6,03 & 5,06 \\
\hline & $33^{\mathrm{f}}$ & $61^{\mathrm{f}}$ & $150^{\mathrm{f}}$ & $131^{\mathrm{f}}$ & $1,14^{\mathrm{f}}$ & 150144 & 1,13 & 0,00 & 9,19 & 0,007 & 0,00 & 13,0 & 0,01 & 0,00 & 9,92 & 0,88 \\
\hline \multirow{6}{*}{2} & $24,6^{\mathrm{c}}$ & $62,4^{c}$ & $352^{c}$ & $244^{\mathrm{c}}$ & $1,45^{\mathrm{c}}$ & 337251 & 1,44 & 10,61 & 4,85 & 0,007 & 15,0 & 7,00 & 0,01 & 4,26 & 2,87 & 0,69 \\
\hline & $26,9^{\mathrm{b}}$ & $60,6^{\mathrm{b}}$ & $300,2^{\mathrm{b}}$ & $255,5^{\mathrm{b}}$ & $1,18^{\mathrm{b}}$ & 300252 & 1,20 & 0,14 & 2,47 & 0,014 & 0,20 & 3,50 & 002 & 0,07 & 1,37 & 1,69 \\
\hline & $28,19^{\mathrm{d}}$ & $80^{\mathrm{d}}$ & $470,3^{\mathrm{d}}$ & $359,3^{\mathrm{d}}$ & $1,31^{\mathrm{d}}$ & 470347 & 1,31 & 0,22 & 8,68 & 0,000 & 0,31 & 12,28 & 0,00 & 0,07 & 3,42 & 0,00 \\
\hline & $28,19^{d}$ & $80^{\mathrm{d}}$ & $462,85^{\mathrm{d}}$ & $359,73^{\mathrm{d}}$ & $1,30^{\mathrm{d}}$ & 470347 & & 5,06 & 9,00 & 0,007 & 7,15 & 12,73 & 0,01 & 1,54 & 3,54 & 0,77 \\
\hline & $30^{\mathrm{e}}$ & $50^{\mathrm{e}}$ & $399,90^{\mathrm{e}}$ & $301,94^{\mathrm{e}}$ & $1,32^{\mathrm{e}}$ & 400302 & 1,38 & 0,07 & 0,04 & 0,042 & 0,10 & 0,06 & 0,06 & 0,03 & 0,02 & 4,55 \\
\hline & $30^{\mathrm{g}}$ & $61^{g}$ & $322,11^{\mathrm{g}}$ & $301,20^{\mathrm{g}}$ & $1,06^{\mathrm{g}}$ & 337302 & 1,13 & 10,53 & 0,57 & 0,049 & 14,89 & 0,80 & 0,07 & 4,62 & 0,27 & 6,60 \\
\hline \multirow{7}{*}{3} & $24,95^{\mathrm{c}}$ & $45^{\mathrm{c}}$ & $618^{c}$ & $406^{\mathrm{c}}$ & $1,52^{\mathrm{c}}$ & 631411 & 1,53 & 9,19 & 3,54 & 0,007 & 13,00 & 5,00 & 0,01 & 2,10 & 1,23 & 0,66 \\
\hline & $25,4^{\mathrm{b}}$ & $69,7^{\mathrm{b}}$ & $481,8^{\mathrm{b}}$ & $340^{\mathrm{b}}$ & $1,42^{\mathrm{b}}$ & 485329 & 1,44 & 2,26 & 7,78 & 0,016 & 3,20 & 11,00 & 002 & 0,66 & 3,24 & 1,55 \\
\hline & $27^{\mathrm{e}}$ & $53^{e}$ & $501,82^{\mathrm{e}}$ & $342,99^{\mathrm{e}}$ & $1,46^{\mathrm{e}}$ & 511370 & 1,44 & 6,49 & 19,10 & 0,014 & 9,18 & 27,01 & 0,02 & 1,83 & 7,87 & 137 \\
\hline & $27,73^{\mathrm{d}}$ & $80^{\mathrm{d}}$ & $799,5^{\mathrm{d}}$ & $573,6^{\mathrm{d}}$ & $1,41^{\mathrm{d}}$ & 799568 & 1,43 & 0,35 & 3,97 & 0,014 & 0,50 & 5,61 & 0,02 & 0,06 & 0,98 & 1,42 \\
\hline & $27,73^{\mathrm{d}}$ & $80^{\mathrm{d}}$ & $799,35^{\mathrm{d}}$ & $562,05^{\mathrm{d}}$ & $1,42^{\mathrm{d}}$ & 799568 & 1,43 & 0,25 & 4,21 & 0,007 & 0,35 & 5,95 & 0,01 & 004 & 1,06 & 0,70 \\
\hline & $29^{\mathrm{e}}$ & $44^{\mathrm{e}}$ & $628,33^{\mathrm{e}}$ & $438,19^{\mathrm{e}}$ & $1,43^{\mathrm{e}}$ & 634438 & 1,44 & 4,01 & 0,13 & 0,007 & 5,67 & 0,19 & 0,01 & 0,90 & 0,04 & 0,70 \\
\hline & $30^{\mathrm{a}}$ & $60^{\mathrm{a}}$ & $648,00^{\mathrm{a}}$ & $417,00^{a}$ & $1,55^{\mathrm{a}}$ & 634412 & 1,53 & 9,90 & 3,54 & 0,014 & 14,00 & 5,00 & 0,02 & 2,16 & 1,20 & 1,29 \\
\hline \multicolumn{3}{|c|}{ Média } & & & & & & 4,31 & 4,76 & 0,02 & 6,09 & 6,73 & 0,02 & 2,38 & 2,94 & 2,16 \\
\hline
\end{tabular}

Fontes: (a) SANTOS (2004); (b) MINAFRA (2007); (c) ZAUK (2008); (d) LIRA et al. (2009); (e) THON et al. (2010); (f) CARVALHO (2006), e (g) LABOISSIÈRE (2008).

Verifica-se que os maiores valores de CR e GP, e os menores de CA são observados nestas faixas de temperatura (Tabela 3). Segundo FUNCK \& FONSECA (2008), mantendo-se a temperatura nos níveis desejáveis e atentando-se para as outras práticas de manejo, consegue-se manter o crescimento das aves em níveis adequados e a uniformidade do lote, além da melhora na CA. Em relação à UR da primeira semana, os melhores CR, GP e CA observados (Tabela 3) estão de acordo com os valores citados na literatura. De acordo com JÁCOME et al. (2007), nos primeiros dias de vida, a UR no galpão deve ser um pouco mais alta, cerca de $70 \%$ para minimizar a desidratação dos pintos e, após este período, de 50-60\% são aceitáveis. De acordo com a Tabela 3, os melhores desempenhos dos pintinhos ocorreram justamente nas URs mais elevadas, confirmando os resultados citados na literatura. 
As médias dos desvios-padrão das variáveis CR, GP e CA foram de 4,31 g; 4,76 g e 0,02 $\mathrm{g} \mathrm{g}^{-1}$, respectivamente, correspondendo aos desvios absolutos de $6,09 \mathrm{~g} ; 6,73 \mathrm{~g} ; 0,02 \mathrm{~g} \mathrm{~g}^{-1}$ e erros percentuais medidos de 2,38; 2,94 e 2,16\% (Tabela 3). De acordo com as regressões lineares simples, com o ajuste da reta passando pela origem, apresentaram $\mathrm{R}^{2}=0,998 ; 0,995 ; 0,976$, para CR, GP e CA, respectivamente (Figura 4). Esses resultados indicaram boa precisão do modelo fuzzy. MEDEIROS (2001), ao criar modelos matemáticos para estimar CR, GP e CA de frangos adultos em função da T, UR e velocidade do ar (V), encontrou valores de $\mathrm{R}^{2}=0,91 ; 0,89$ e 0,72 , respectivamente. Esses valores de $\mathrm{R}^{2}$ mais elevados obtidos por meio do sistema de inferência fuzzy, em relação ao modelo criado por MEDEIROS (2001), permitiram que a predição das respostas produtivas: CR, GP e CA fossem mais realísticas. Dessa forma, os resultados encontrados neste trabalho indicam ser possível a estimativa de GP, CR e CA em função das variáveis climáticas e da idade das aves por meio de modelos fuzzy. A operacionalização desses resultados ajuda no suporte à decisão do controle da climatização do galpão, garantindo, assim, melhor produção.

Ao se compararem os valores de GP simulados pelo modelo fuzzy com aqueles obtidos experimentalmente na granja comercial de criação de frangos de corte em estudo (Figura 5), verificou-se que os valores de desvio-padrão, desvio absoluto, erro percentual e coeficiente de determinação $\left(\mathrm{R}^{2}\right)$ foram de $14,75 \mathrm{~g}, 20,86 \mathrm{~g}, 11,98 \%$ e 0,9753 , respectivamente. Esses resultados indicam que o modelo fuzzy proposto teve precisão adequada para a predição do GP das aves.

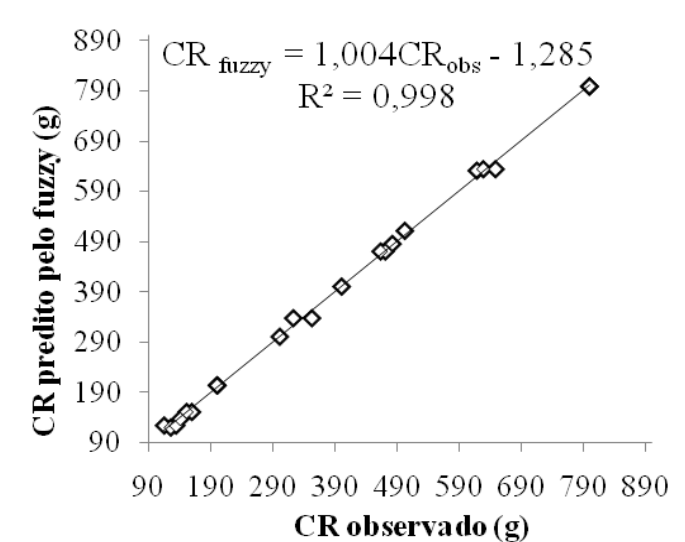

(a)

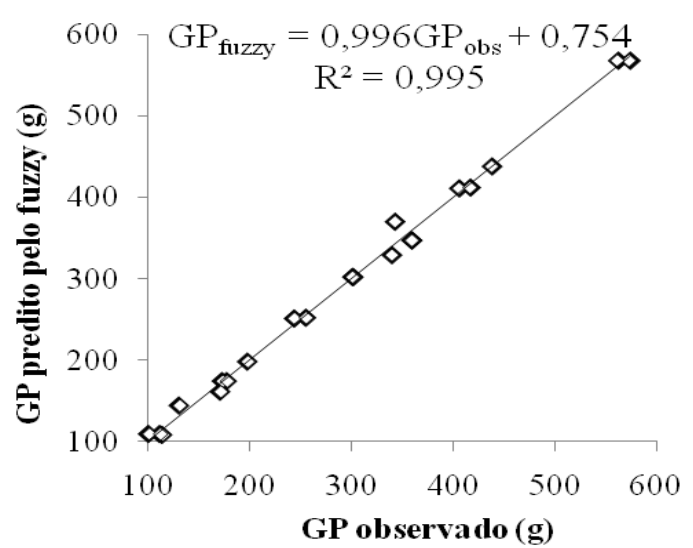

(b)

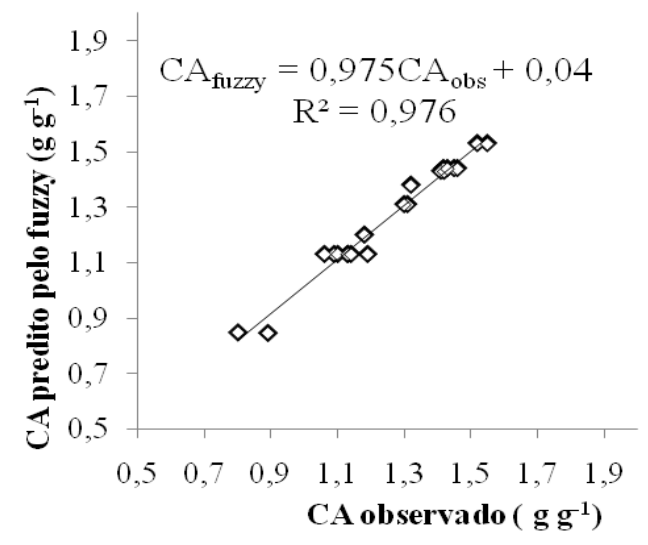

(c)

FIGURA 4. Regressões lineares para as variáveis de saída: (a) consumo de ração (CR); (b) ganho de peso (GP); (c) conversão alimentar (CA), em função dos valores preditos pela lógica fuzzy e os valores medidos pela literatura. Linear regressions for the output variables (a) feed intake (CR), (b) weight gain (GP), (c) feed conversion (CA) according to the values predicted by the fuzzy logic and the values measured by the literature. 
As temperaturas medianas na primeira, segunda e terceira semanas de vida das aves foram de $31,3{ }^{\circ} \mathrm{C}, 28,5{ }^{\circ} \mathrm{C}$ e $28,3{ }^{\circ} \mathrm{C}$, respectivamente (Figura 6A). Apesar de os valores medianos da temperatura do ar estarem contidos nas faixas de temperatura consideradas ideais para cada semana previamente citada, que são de $32{ }^{\circ} \mathrm{C}$ a $34{ }^{\circ} \mathrm{C} ; 28{ }^{\circ} \mathrm{C}$ a $32{ }^{\circ} \mathrm{C}$; e $26{ }^{\circ} \mathrm{C}$ a $28{ }^{\circ} \mathrm{C}$, respectivamente (OLIVEIRA et al., 2006), verifica-se que, durante 86,6; 55 e 76,7\% do tempo (Figura 6C), as aves estavam sujeitas a temperaturas fora das faixas de conforto.

De acordo com MOURA et al. (2010) e SILVA et al. (2009), valores de temperaturas superiores ou inferiores às faixas consideradas ideais ao frangos de corte, de acordo com a idade, prejudicam o desempenho e a produção de aves.

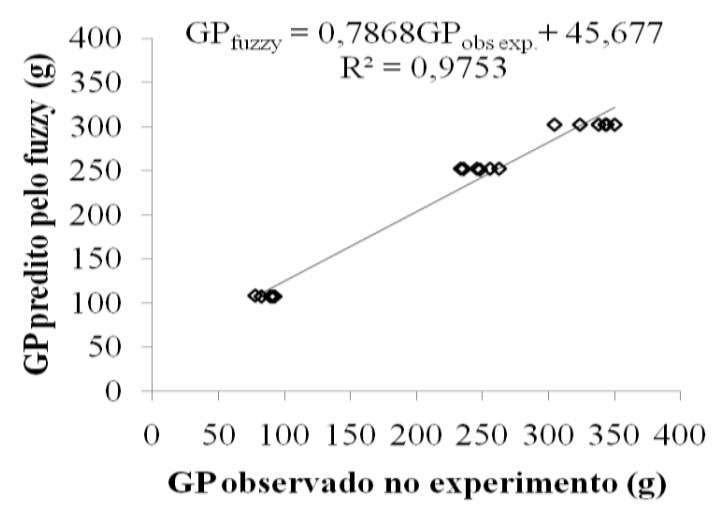

FIGURA 5. Regressão linear para o ganho de peso (GP), em função dos valores preditos pela lógica fuzzy e os valores medidos em experimentação no campo. Linear regression for the weight gain (GP) as a function of the values predicted by the fuzzy logic and the values measured in the field experiment.

Com relação à UR, os valores medianos de 61; 63 e 64\% foram observados para a primeira, segunda e terceira semanas, respectivamente (Figura 6B). Ao se analisarem as frequências de ocorrência de UR para as três semanas em questão, verifica-se que, durante 38,5; 45,4 e 40,9\% do tempo (Figura 6D), as aves estavam sujeitas a UR fora das faixas de conforto para cada semana de criação. Dentre as possíveis implicações aos animais, podem-se citar o comprometimento do balanço da homeotermia (MOURA et al., 2010), podendo causar a desidratação dos animais, a redução do desempenho produtivo, dentre outras respostas indesejáveis.

Predições de GP com o modelo fuzzy mostraram que, se as aves fossem criadas em condições ideais de T e UR durante as 3 semanas de criação, o GP médio semanal seria de 0,117; 0,298 e $0,370 \mathrm{~kg}$, respectivamente, enquanto nos galpões avaliados observaram-se os valores de 0,085; 0,247 e 0,334 kg. Considerando que nos dois galpões estavam alojados 56 mil aves, o adicional de GP por ave, em cada semana de vida, seria de 0,$031 ; 0,051$ e $0,036 \mathrm{~kg}$, o que resultaria no aumento de $6.664 \mathrm{~kg}$ de peso vivo dos animais aos 21 dias de alojamento. 
A

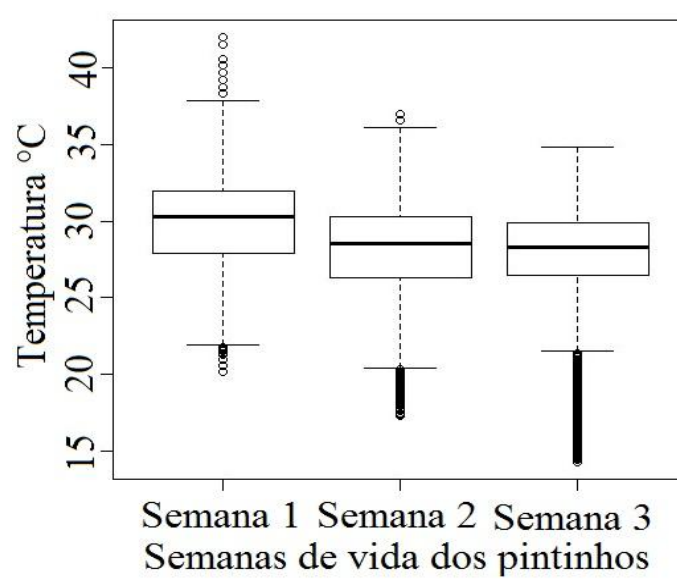

C

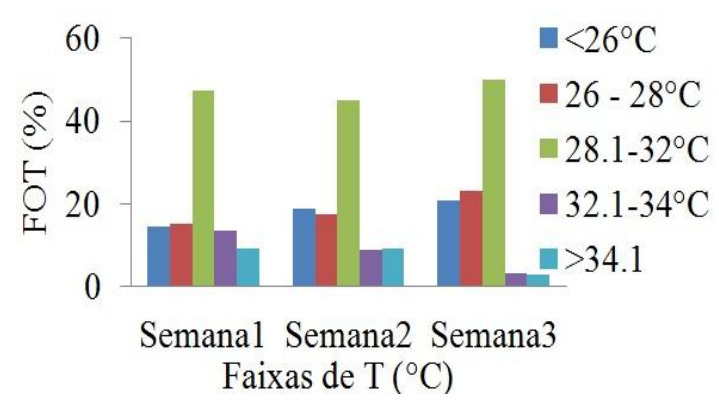

B

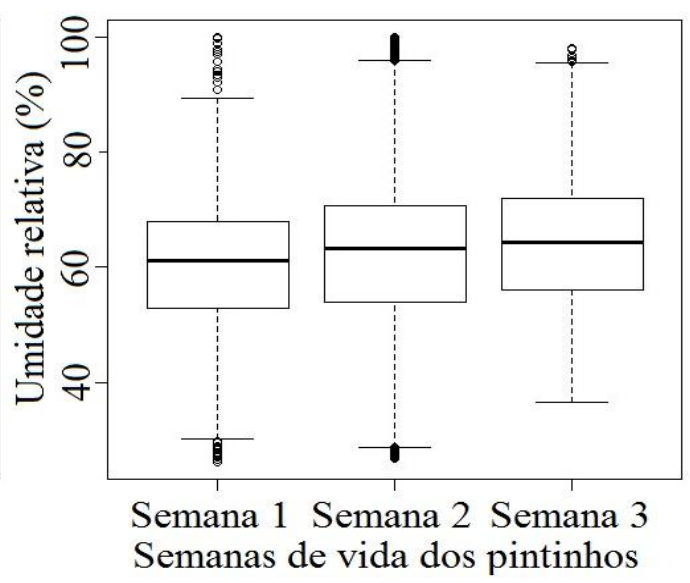

$\mathrm{D}$

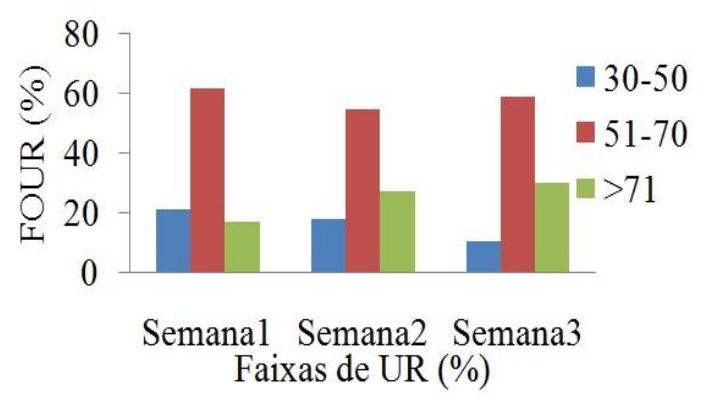

FIGURA 6. Box-plot e frequência de ocorrências de temperaturas (FOT, \%) (A e C, respectivamente) e umidades relativas (FOUR, \%) (B e D, respectivamente) nas três primeiras semanas de vida da ave. Box-plot and frequency of occurrence of temperatures (FOT, \%) (A and C, respectively) and relative humidities (FOUR, $\%$ ) (B and D, respectively) in the first three weeks of the bird's growth.

\section{CONCLUSÕES}

O desenvolvimento do modelo fuzzy, em função das variáveis climáticas e idades das aves, mostrou-se adequado para a predição do consumo de ração, ganho de peso e conversão alimentar de frangos de corte com idade entre 1 a 21 dias, caracterizando-se como ferramenta de suporte à tomada de decisão no controle do acondicionamento térmico de galpões avícolas, garantindo, assim, melhor produção.

\section{AGRADECIMENTOS}

Os autores expressam seus agradecimentos à CAPES, CNPq e FAPEMIG, pelo suporte financeiro a esta pesquisa.

\section{REFERÊNCIAS}

ABREU, P.G. de; BAÊTA, F.C.; ABREU, V.M.N.; SOARES, P.R.; PERDOMO, C.C.; SILVA, M.A. Desempenho produtivo e bioeconômico de frangos de corte criados em diferentes sistemas de aquecimento. Revista Brasileira de Zootecnia, Viçosa-MG, v.29, n.1, p.159-167, 2000.

AMENDOLA, M.; CASTANHO, M. J.; NÄÄS, I. A.; SOUZA, A. L. Análise matemática de condições de conforto térmico para avicultura usando a teoria dos conjuntos Fuzzy. Biomatemática Brasil, Campinas, v.14, p.87-92, 2004. 
AMENDOLA, M.; SOUZA, A.L.; BARROS, L.C. Manual do uso da teoria dos conjuntos fuzzy no MATLAB 6.5. Biomatemática Brasil, Campinas, v.1, p.1-46, 2005.

AYYUB, B. M.; KLIR, G. J. Uncertainty Modeling and Analysis in Engineering and the Sciences. Boca Raton: Chapman \& Hall/CRC, 2006. 378 p.

CADENAS, J.M.; PELTA, D.A.; PELTA, H.R.; VERDEGAY, J.L. Application of Fuzzy optimization to diet problems in Argentinean farms. European Journal of Operational Research, Amsterdam, v.158, n.1, p.218-228, 2004.

CARVALHO, J.C.C. Complexos enzimáticos em rações fareladas para frangos de corte. 2006. $64 \mathrm{f}$. Dissertação (Mestrado em Zootecnia) - Universidade Federal de Lavras, Lavras, 2006.

CORDEIRO, M.B.; TINÔCO, I. de F. F.; SILVA, J.N da; VIGODERIS, R.B.; PINTO, F. de A. de C.; CECON, P.R. Conforto térmico e desempenho de pintos de corte submetidos a diferentes sistemas de aquecimento no período de inverno. Revista Brasileira de Zootecnia, Viçosa-MG, v.39, n.1, p.217-224, 2010.

CORNELISSEN, A.M.G.; VAN DEN BERG, J.; KOOPS, W.J.; AND KAYMAK, U. Eliciting expert knowledge for fuzzy evaluation of agricultural production systems. Rotterdam: Erasmus Research Institute of Management, 2002. (Report, ERS-2002-108-LIS). Disponível em: http://papers.ssrn.com/sol3/papers.cfm?abstract_id=371055. Acesso em: 20 dez. 2011.

FUNCK, S.R.; FONSECA, R. A. Avaliação energética e de desempenho de frangos com aquecimento automático a gás e a lenha. Revista Brasileira de Engenharia Agrícola e Ambiental, Campina Grande, v.12, n.1, p.91-97, 2008.

JACOME, I.M.T.D.;FURTADO, D.A.; LEAL, A.F.; SILVA, J.H.V.; MOURA, J.F.P. Avaliação de índices de conforto térmico de instalações para poedeiras no Nordeste do Brasil. Revista Brasileira de Engenharia Agrícola e Ambiental, Campina Grande, v.11, n.5, p.527-531, 2007.

KLIR G.J.; YUAN B. Fuzzy sets and fuzzy logic: theory and applications. New Jersey: Prentice Hall, PRT, 1995. 563 p.

LABOISSIÈRE, M. Farinhas de resíduos de abatedouro avícolas em diferentes graus de processamento em rações pré-iniciais e iniciais de frangos de corte. $2008.70 \mathrm{f}$. Dissertação (Mestrado em Medicina Veterinária) - Universidade Federal de Goiás, Goiânia, 2008.

LIRA, R.C.; RABELLO, C.B.V.; FERREIRA, P.V.; LANA, G.R.Q.; LÜDKE, J.V.; DUTRA JÚNIOR, W.M. Inclusion of guava wastes in feed for broiler chickens. Revista Brasileira de Zootecnia, Viçosa-MG, v.38, n.12, p.2.401-2.407, 2009.

MANAGEMENT GUIDE BROILER. 2006. Disponível em: http://www.hubbardbreeders. com/managementguides/index.php?product=5. Acesso em: 7 dez. 2009.

MARCHINI, C.F.P.; SILVA, P.L.; NASCIMENTO, M.R.B.M.; BELETTI, M.E.; GUIMARÃES, E.C.; SOARES, H.L. Intestinal morphometry of the duodenal mucosa in broiler chickens underwent to high cyclic environment temperature. Arquivo Brasileiro de Medicina Veterinária e Zootecnia, Belo Horizonte, v.61, n.2, p.491-497, 2009.

MEDEIROS, C.M. Ajuste de modelos e determinação de índice térmico ambiental de produtividade para frangos de corte. 2001. 115 f. Tese (Doutorado em Engenharia Agrícola) - Universidade Federal de Viçosa, Viçosa, 2001.

MENEZES, A.G.; NÄÄS, I.A.; BARACHO, M.S. Identification of critical points of thermal environment in broiler production. Revista Brasileira de Ciência Avícola, Campinas, v.12, n.1, 2010 .

MINAFRA, C.S. Produção e suplementação com $\alpha$-amilase de Cryptococcus flavus e Aspergillus Níger HM2003 na dieta de frangos de corte de um a 21 dias de idade. 2007. $141 \mathrm{f}$. Tese (Doutorado em Bioquímica Agrícola) - Universidade Federal de Viçosa, Viçosa, 2007. 
MOURA, D.J.; BUENO, L.G.F.; LIMA, K.A.O de; CARVALHO, T.M.R. de; MAIA, A.P.A.M. Strategies and facilities in order to improve animal welfare. Revista Brasileira de Zootecnia, Viçosa, v.39, p.311-316, 2010. Supl. Especial.

MOURA, D.J.; NÄÄS, I.A; ALVES, E.C. de S.; CARVALHO, T.M.R de; VALE, M.M. do; LIMA, K.A.O de. Análise de ruído para a avaliação do conforto térmico de pintinhos. Scientia Agricola, Piracicaba, v.65, n.4, p.438-443, 2008.

MOURA, D.J.; NÄÄS, I. A.; PEREIRA, D. F.; SILVA, R. B.T. R.; CAMARGO, G. A. Animal welfare concepts and strategy for poultry production: a review. Revista Brasileira de Ciência Avícola, Campinas, v.8, n.3, p.137-148, 2006.

NÄÄS, I. A.; QUEIROZ, M. P. G.; MOURA, D. J de; BRUNASSI, L. A. Estimativa de estro em vacas leiteiras utilizando métodos quantitativos preditivos. Ciência Rural, Santa Maria, v.38, n.8, p.2.383-2.387, 2008.

NATIONAL RESEARCH COUNCIL. Nutrient requirements of poultry. Washington, 1994. $151 \mathrm{p}$.

NAZARENO, A.C.; PANDORFI, H.; ALMEIDA, G.L.P.; GIONGO, P.R.; PEDROSA, E.M.R.; GUISELINI, C. Avaliação do conforto térmico e desempenho de frangos de corte sob regime de criação diferenciado. Revista Brasileira de Engenharia Agrícola e Ambiental, Campina Grande, v.13, n.6, p.802-808, 2009.

OLIVEIRA, H.L.; AMENDOLA, M.; NÄÄS, I. A. Estimativa das condições de conforto térmico para avicultura de postura usando a teoria dos conjuntos fuzzy. Engenharia Agrícola, Jaboticabal, v.25, n.2, p.300-307, 2005.

OLIVEIRA, R.F.M.; DONZELE, J.L.; ABREU, M.L.T.; FERREIRA, R.A.; VAZ, R.G.M.V.; CELLA, P. S. Efeitos da temperatura e da umidade relativa sobre o desempenho e o rendimento de cortes nobres de frangos de corte de 1 a 49 dias de idade. Revista Brasileira de Zootecnia, ViçosaMG, v.35, n.3, p.797-803, 2006.

OWADA, A.N.; NÄÄS, I. A.; MOURA, D. J.; BARACHO, M.S. Estimativa de bem-estar de frangos de corte em função da concentração de amônia e grau de luminosidade no galpão de produção. Engenharia Agrícola, Jaboticabal, v.27, n.3, p.611-618, 2007.

PANDORFI, H.; SILVA, I. J. O.; GUISELINI, C.; PIEDADE, S.M.S.P. Uso da lógica fuzzy na caracterização do ambiente produtivo para matrizes gestantes. Engenharia Agrícola, Jaboticabal, V.27, n.1, p.83-92, 2007.

PEREIRA, D.F.; BIGHI, C.A.; GABRIEL FILHO, L.R.; GABRIEL, C.P.C. Sistema fuzzy para estimativa do bem-estar de matrizes pesadas. Engenharia Agrícola, Jaboticabal, v.28, n.4, p.624634, 2008.

PERISSINOTTO, M.; MOURA, D.J.; CRUZ, V. F. da; SOUZA, S.R.L. de; LIMA, K.AO de; MENDES, A.S. Conforto térmico de bovinos leiteiros confinados em clima subtropical e mediterrâneo pela análise de parâmetros fisiológicos utilizando a teoria dos conjuntos fuzzy. Ciência Rural, Santa Maria, v.39, n.5, p.1.492-1.498, 2009.

SALGADO, D.D.; NÄÄS, I. A.; PEREIRA, D.F.; MOURA, D.J. Modelos estatísticos indicadores de comportamentos associados a bem-estar térmico para matrizes pesadas. Engenharia Agrícola, Jaboticabal, v.27, n.3, 2007.

SANTOS, A.L. dos. Potencial de crescimento, desempenho, rendimento de carcaça e qualidade da carne de diferentes linhagens de frango de corte convencional e tipo caipira. 2004. $95 \mathrm{f}$.

Dissertação (Mestrado em Zootecnia) - Faculdade de Ciências Agrárias e Veterinárias, Universidade Estadual Paulista, 2004.

SANTOS, R.C.; NÄÄS, I.A.; MOREIRA, F.R.; GOMES FILHO, R. Aplicação da lógica nebulosa na simulação de estro de vacas leiteiras. Enciclopédia Biosfera, Goiânia, v.5, n.8, p.1-6, ago.2009. 
SILVA, V. K.; SILVA, J.D.T.; GRAVENA, R.A.; MARQUES, R.H.; HADA, F.H.; MORAES, V.M.B. m. Revista Brasileira de Zootecnia, Viçosa-MG, v.38, n.4, 2009.

TAYLOR, J. Delphi method applied to tourism. In: WITTIS, M. L. Tourism marketing and management handbook. New York: Prentice Hall, 1988. 95-99 p.

TEIXEIRA, E N.M.; SILVA, J.H.V.; COSTA, F.G.P.; MARTINS, T.D.D.; GIVISIEZ, P.E.N.; FURTADO, D.A. Efeito do tempo de jejum pós-eclosão, valores energéticos e inclusão do ovo desidratado em dietas pré-iniciais e iniciais de pintos de corte. Revista Brasileira de Zootecnia, Viçosa-MG, v.38, n.2, 2009.

THON, M.S.; STRINGHINI, J.H.; JARDIM FILHO, R.M.; ANDRADE, M.A.; CAFÉ, M.B.; LEANDRO, N.S.M. Níveis de proteína e de arginina digestível na ração pré-inicial de frangos de corte. Revista Brasileira de Zootecnia, Viçosa-MG, v.39, n.5, p.1.105-1.111, 2010.

TOLON, Y.B.; BARACHO, M.S.; NÄÄS, I.A.; ROJAS, M.; MOURA, D.J. Ambiências térmica, aérea e acústica para reprodutores suínos. Engenharia Agrícola, Jaboticabal, v.30, n.1, p.1-13, 2010. VIGODERIS, R.B.; CORDEIRO, M.B.; TINÔCO, I.F.F.; MENEGALI, I.; SOUZA JÚNIOR, J.P.; HOLANDA, M.C.R. Avaliação do uso de ventilação mínima em galpões avícolas e de sua influência no desempenho de aves de corte no período de inverno. Revista Brasileira de Zootecnia, Viçosa-MG, v.39, n.6, 2010.

ZAUK, N.H.F. Desempenho produtivo, características de carcaças e morfometria intestinal de frangos de corte recebendo dietas pré-iniciais com inclusão de diferentes níveis de extrato de levedura (Saccharmyces cerevisiae). 2008.140 f. Tese (Doutorado em Nutrição Animal) Faculdade de Agronomia Eliseu Maciel, Universidade Federal de Pelotas, Pelotas, 2008. 